Journal of

Case Reports: Open Access

\title{
Rare Seen Bilateral Orbital Chloroma
}

\section{Serkan Ünlï̈, Mehtap Ilgar, Mehmet Akçiçek}

Malatya Education and Research Hospital, Radiology Department, Medical Doctor, Malatya-Turkey

${ }^{\star}$ Corresponding author: Serkan Ünlü, Malatya Education and Research Hospital, Radiology Department, Medical Doctor, Malatya-Turkey; Tel: +5066285899; E-mail:serkanunlu19@yahoo.com

Received Date: December 24, 2019 Accepted Date: January 09, 2020 Published Date: January 11, 2020

Citation: Serkan Ünlü (2020) Rare Seen Bilateral Orbital Chloroma. Case Reports: Open Access 5: 1-4.

\begin{abstract}
Orbital chloroma (OK), also known as granulocytic sarcoma (GS), occurs in the form of focal masses in the bone and soft tissues of immature myeloid cells in the bone marrow. Leukemia is a rare form of involvement (1). Granulocytic sarcoma, chronic myeloid leukemia (CML), polycythemia vera, myelofibrosis, and chronic eosinophilic leukemia may be seen, although acute myeloid leukemia is usually seen (2). We aimed to present Magnetic Resonance Imaging (MRI) findings of our case of bilateral orbital chloroma with CML disease.
\end{abstract}

Keywords: Orbita; Chloroma; Mri

\section{Material and Method}

Coronal T1WI, Axial T1WI SPIR, Axial T2WI, Axial T2WI SPIR, Axial DWI, and Axial T1WI SPIR contrast sequences were obtained with MRI in a follow-up CML-guided patient.

\section{Radiological Findings}

Coronal T1WI (Figure 1) in the patient with CML diagnosis shows the lateral orbital lateral wall of bilateral orbit superior to the sphenoid bone mass and the right intracoronary and extraconal left to the left in the extraconal area gray matter and circumferential muscle tissue and isointense solid locoregional lesions are seen.
T1WI SPIR (Figure 2), there is no suppression due to a lack of fat content in the lesions and isointense with gray matter.

In T2WI (Figure 3), retrobulbar fat is an isointense with the hypointense gray matter according to the tissue.

In the T2WI SPIR (Figure 4), the lesions are oil-free and are isointense with gray matter. Diffusion restriction is observed in DWI lesions (figure 5)

In contrast T1WI SPIR (Figure 6), the contrast enhancement is observed homogeneously in the middle of the lesions. 


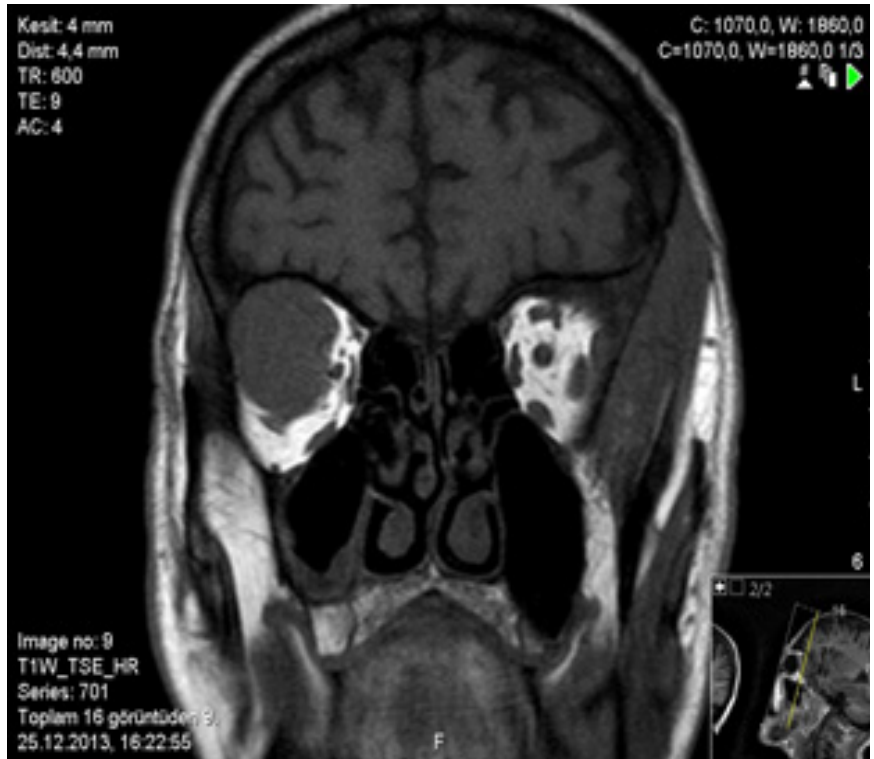

Figure 1: Bilateral orbital superiority in coronal T1WI is seen in the lateral aspect of the orbital and on the right side holding the large canal of the sphenoid bone and intraconal and extraconal left in the extracorporeal area gray matter and circumferential muscle tissue and isointense solid lesion.

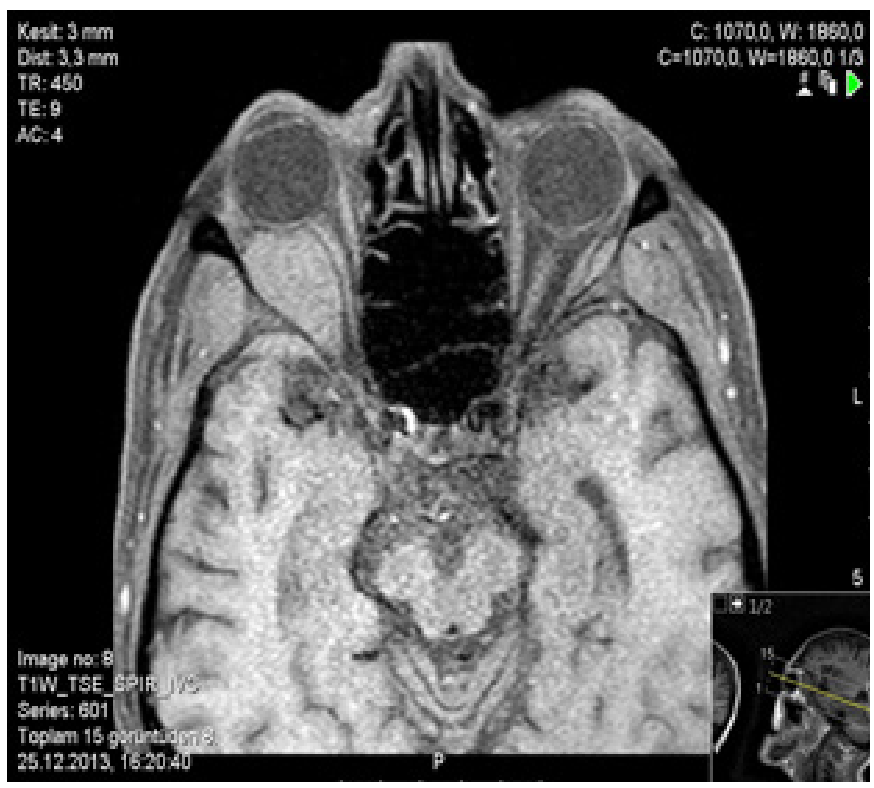

Figure 2: In T1WI SPIR, there is no suppression due to lack of fat content in the lesions and isointense with gray matter

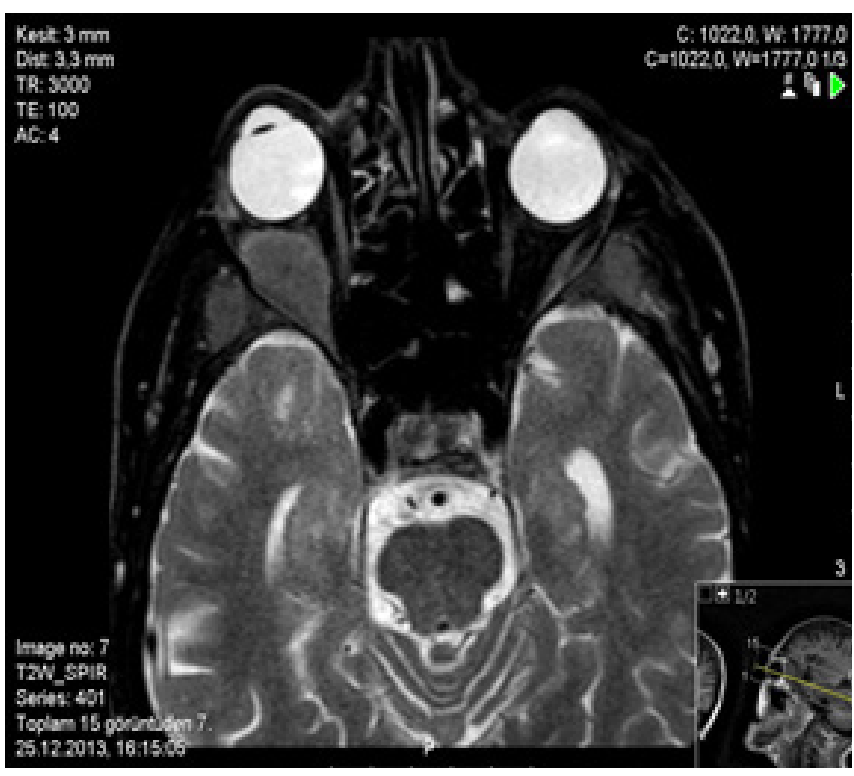

Figure 3: In T2WIretro bulbar fat is seen as isointense with the hypointense gray matter according to tissue

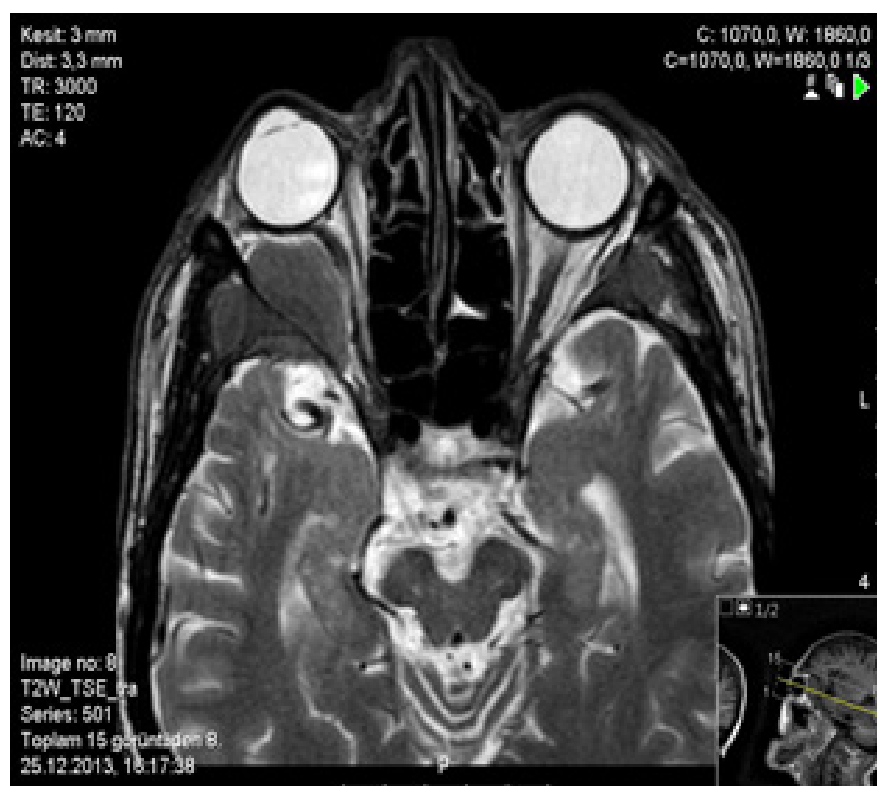

Figure 4: T2WIin the lesion does not contain oil in SPIR and is isointense with gray matter. 


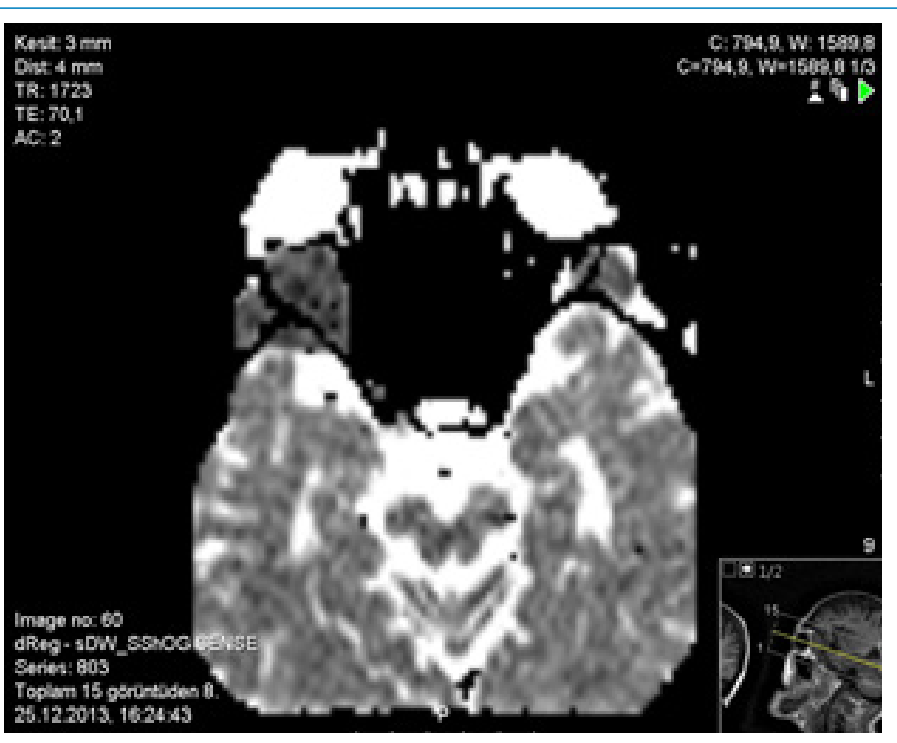

Figure 5:Diffusion restriction is observed in lesions.

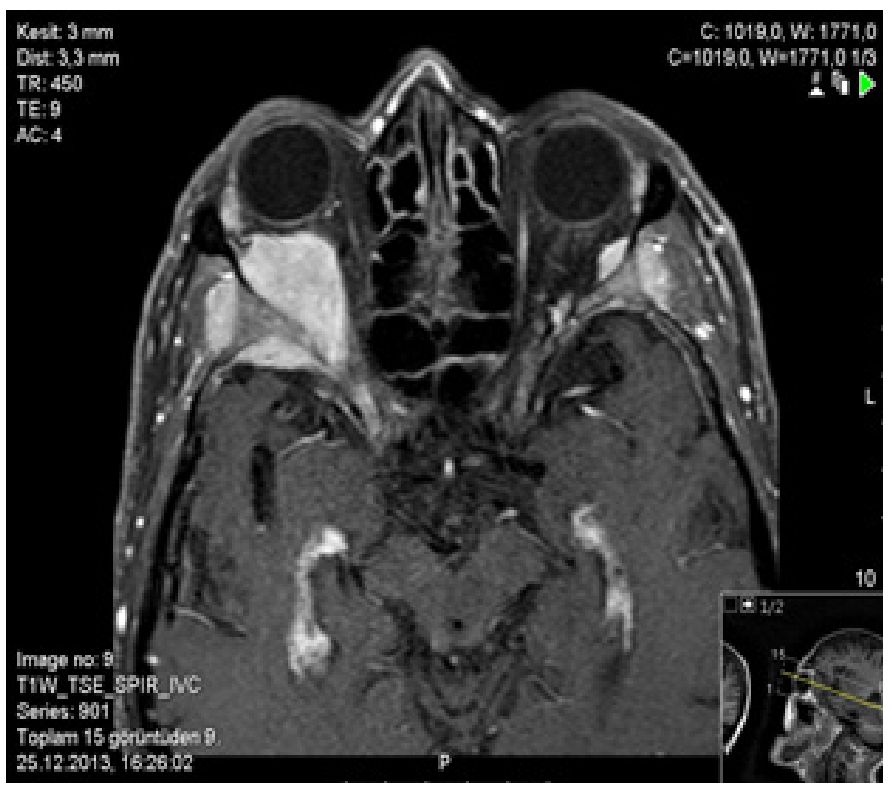

Figure 6: Contrast-enhanced T1WI SPIR shows a homogeneous contrast enhancement in the middle of the lesions.

\section{Discussion}

Orbital Chloroma (OC) is known as granulocytic sarcoma (GS) and focal masses in bone and soft tissues formed by immature myeloid cells in the bone marrow. Leukemia is a rare form of involvement [1]. Granulocytic sarcoma chronic myeloid leukemia (CML), polycythemia rubra vera, myelofibrosis, and chronic eosinophilic leukemia may also be seen, although acute myeloid leukemia is usually seen. The OC intraconal or extraconal area may be bilateral or unilateral, homogeneous, well defined, extending to the adjacent bone and surrounding structures.
OC brain or muscle is hypointense or isointense in T1WI and shows heterogeneous isointense or hyperintense heterogeneous signal in T2WI and homogenous post-contrast enhancement in T2WI $[2,3]$. OC bone and sclera are rarely destructive to the bone. In our case, there was no destruction in the bones. OC can more orbital lateral wall, sphenoid bone large canal and bilateral petrous apex [4]. In the differential diagnosis of a patient known to be leukemia, abscess, hematoma, and secondary malignancies can be considered. When the abscissa shows ring-shaped enhancement, OC also shows homogeneous contrast and the hematoma shows no enhancement. When OC is bilateral, Langerhans cell histiocytosis (LHH) and metastatic neuroblastoma (MNB) may be considered in the differential diagnosis.

Ocular adnexal lymphomas involve the orbit, eyelid, lacrimal gland, and conjunctiva [5]. Myeloid sarcoma is a rare, extra-medullary presentation that can occur as an isolated tumor, concurrently with or at relapse of acute myeloid leukemia [6].

The presence of lytic lesions in other bones in LHH and MNB allows differentiation from OCD. Diffusion-weighted imaging (DWI) shows abscess diffusion restriction [3]. In our case, there is a restriction on the DWI in the OC. Therefore; the contrast sequences are more useful in distinguishing abscess OC. Approximately $60 \%$ of cases arise without specific gender preferences in children under 15 years of age. The reported 2-9\% incidence of acute myeloid leukemia (AML) is an unusual occurrence [7].

Before diagnosing underlying leukemia, it is well known that AML could first be viewed with orbital involve. Leukemia's softer tissue accumulations were previously called granulocytic sarcoma or chloroma [8]. Clinical presentation with orbital involvement most commonly is with proptosis. Other symptoms like periorbital cellulitis or swelling or mass in the lacrimal gland or eyelid may be seen. It may be bilateral. Most of the reported cases had decreased visual acuity and restricted extra-ocular movements [9].

In the end, in patients with acute myeloid leukemia with associated bone marrow disease, granulocytic sarcoma usually appears in adult patients as extramedullary relapse. Clinical presentations are complex and may include signs of compression or obstruction. Given the complex nature of the disease, there are a few characteristics of granulocytic sarcoma in the central nervous system that may help to differentiate these lesions from other severe complications of leukemia. Multiple, enhanced, sol- 
id mass in different places in a patient with acute myeloid leukemia or myeloproliferative or myelocytic disorders are strongly suggestive signs of granulocytic sarcomas [10].

\section{Results}

MR imaging helps in better characterization of the soft tissue components of the orbit. Orbital Chloroma is seen more orbital region in the head and neck region and shows well restricted homogeneous contrast enhancement and shows diffusion limitation in DAG. Orbital Chloroma is considered in the diagnosis of solid soft-tissue mass observed in the subperiosteal area of the orbital region in patients with leukemia diagnosis and MRI can be used to distinguish it from other masses. Nevertheless, in these patients, imaging alone is insufficient, and aspiration biopsies are warranted to exclude the possibility of an abscess.

\section{References}

1. Uyesugi WY1, Watabe J, Petermann G (2000) Orbital and facial granulocytic sarcoma (chloroma): a case report, Pediatric Radiol 30: 276-278.

2. Noh B W, Park S W, Chun J E, et al. (2009) Granulocytic Sarcoma in the Head and Neck: CT and MR Imaging Findings, Clinical and Experimental Otorhinolaryngology 2: 66-71.

3. Youssef A, Raafat T, Mahfouz Y, et al. (2014) Orbital manifestation of the Hematopoietic Tumors: Imaging Features and Differential Diagnosis, ECR.

4. Xian J, Wang Z, He L (2006) Characteristic MRI findings of metastatic neuroblastomas and chloramines in the orbit in children, Proc. intl.soc. Mag. Reson. Med. 14 .

5. Sahu KK, Uthamalingam P, Sampath S, Jinagal J, Das A, et al. (2014) Ocular adnexal lymphomas: report of 2 cases of mantle cell lymphomas and short review of the literature. Indian J Hematol Blood Transfus 30: 163-168.

6. Sahu KK, Uthamalingam P, Sampath S, Jinagal J, Das A, et al. ( 2014) Ocular adnexal lymphomas: report of 2 cases of mantle cell lymphomas and short review of the literature. Indian J Hematol Blood Transfus 30: 163-168.

7. Guermazi A, Feger C, Rousselot P, Merad M, Benchaib N, Bourrier P, et al. (2002) Granulocytic sarcoma (chloroma): imaging findings in adults and children. AJR Am J Roentgenol 178: 319-325.
8. Jha BK, Lamba PA (1971) Proptosis as a manifestation of acute myeloid leukemia. Br J Ophthalmol 55844- 55847.

9. Murthy R, Vemuganti GK, Honavar SG, Naik M, Reddy V (2009) Extramedullary leukemia in children presenting with proptosis. J Hematol Oncol. 24; 2:4.

10. GC Ooi, CS Chim, PL Khong, et al. (2001) Radiologic Manifestations of Granulocytic Sarcoma in Adult Leukemia, American Journal of Roentgenology 176: 1427-1431. 